\title{
Consumo de Álcool na Adolescência e Relação com Uso Excessivo de Bebidas Alcóolicas dos Pais: Estudantes de Quatro Escolas de Porto Alegre
}

\author{
Alice Rodrigues Willhelm \\ João Carlos Centurion Cabral \\ Jana Oliveira Steiger \\ José Felipe Flores da Silva \\ Luiza Mugnol Ugarte \\ Rosa Maria Martins de Almeida \\ Universidade Federal do Rio Grande do Sul \\ Porto Alegre, RS, Brasil
}

\begin{abstract}
RESUMO
O objetivo geral do estudo foi investigar a relação entre o consumo de álcool pelos adolescentes com a percepção dos mesmos sobre a quantidade de bebidas alcoólicas ingeridas pelos pais. A amostra foi composta de 74 adolescentes estudantes com idade entre 10 e 16 anos, de escolas públicas e privadas de Porto Alegre, RS. Foi aplicado um questionário sobre o primeiro uso de álcool e drogas. Verificou-se associação estatisticamente significativa entre considerar que alguém na família bebe excessivamente e qual familiar tem tal comportamento $\left(\chi^{2}=74 ; \mathrm{gl}=4\right.$; $p<0,001) .74 \%$ dos adolescentes de 15 e 16 anos já experimentaram algum tipo de bebida alcoólica. Foi possível observar relação significativa entre considerar que um familiar bebe excessivamente e o consumo do álcool pelo adolescente. Concluiu-se que adolescentes que consideram que seus familiares bebem em demasia, consomem álcool mais precocemente, com mais intensidade e bebidas com percentual alcóolico maior.
\end{abstract}

Palavras-chave: Adolescência; Álcool; Família.

\section{ABSTRACT}

Alcohol Consumption in Adolescence and the Relationship with Alcoholic Beverage Excessive Use of Parents: Students of Four Schools of Porto Alegre

The overall objective of this study was to investigate what is the relationship between the perception of teens about how their parents consume alcoholic beverages. The sample consisted of 74 adolescent students aged between 10 and 16 years of public and private schools in Porto Alegre, RS. A questionnaire about the first use of alcohol and drugs was applied. We have seen that there is significant association between considering someone in the family drinks too much and wich relative has such behavior $\left(\chi^{2}=74, \mathrm{~g} 1=4, p<0.001\right) .74 \%$ of teenagers aged 15 to 16 said they had experienced some type of alcoholic beverage. Moreover, we observed a significant relationship between the results to consider a relative drinks excessively and alcohol consumption by adolescents. This study concluded that adolescents who considered your parents drink much alcohol drink earlier, with more intensity and beverage with more alcohol.

Keywords: Adolescence; Alcohol; Family.

\section{RESUMEN}

Consumo de Alcohol de los Adolescentes y Relación con el Uso Excesivo de Bebidas Alcohólicas Padres: Estudiantes de Cuatro Escuelas de Porto Alegre

El objetivo general del estudio fue investigar la relación entre el consumo de alcohol por los adolescentes con su percepción acerca de la cantidad de alcohol ingerida por los padres. La muestra fue composta por 74 adolescentes estudiantes de edades comprendidas entre 10 y 16 años de escuelas públicas y privadas de Porto Alegre, RS. Se aplicó un cuestionario en el primer uso de alcohol y drogas. Hubo asociación estadísticamente significativa entre considerar a alguien en las bebidas de la familia también familiares y que tiene tal comportamiento $\left(\chi^{2}=74, \mathrm{~g} 1=4, p<0.001\right) .74 \%$ de los adolescentes entre 15 y 16 años han experimentado algún tipo de bebida alcohólica. Se observó una relación significativa entre la familia considera que un consumo de alcohol y el consumo en exceso de los adolescentes. Se concluyó que los adolescentes que sienten que sus familias consumen beber demasiado, consumen alcohol antes, con más intensidad y las bebidas alcohólicas con un mayor porcentaje.

Palabras clave: Adolescencia; Alcohol; Familia. 


\section{INTRODUÇÃO}

O período da adolescência é demarcado por comportamentos de risco e atos impulsivos, caracterizado também por ausência do controle inibitório (Shirtcliff, Dahl, \& Pollak, 2009; Crone, 2009). Esses comportamentos são frequentemente relacionados com o uso ou abuso de substâncias, atividades criminosas e sexo desprotegido (Gullo \& Dawe, 2008; VerdejoGarcia, Lawrence, \& Clark, 2008). Muitos estudos buscam encontrar quais são os principais fatores que influenciam os adolescentes a começarem a usar drogas (Benchaya, Bisch, Moreira, Ferigolo, \& Barros, 2011; Janssen, Mathijssen, Bon-Martens, Oers, \& Garretsen, 2014; Malta, Mascarenhas, Porto, Barreto, \& Neto, 2014; Roehrs, Lenardt, \& Maftum, 2008). Dentre esses, diversos pontuam que o papel familiar é um fator crucial na decisão do jovem de consumir ou não álcool (Haugland, Holmen, Ravndal, \& Bratberg, 2013; Loke \& Mak, 2013; Looze et al., 2014).

Existem algumas atitudes na relação pais e adolescentes que podem influenciar os resultados de desenvolvimento do adolescente, assim como podem frear ou estimular o comportamento de risco (Skinner, Johnson, \& Snyder, 2005; Newman, Harrison, Dashiff, \& Davies, 2008). Estudo mostrou que afeição, apoio e conexão familiar são fatores que protegem os filhos de tais comportamentos de risco (Loke e Mak, 2013). Os que possuem menos supervisão ou suporte dos pais têm maior tendência de usar álcool antes da idade legal se comparados com os que são mais supervisionados (Loke \& Mak, 2013). Os adolescentes mais suscetíveis a consumir álcool são os que faltam às aulas sem o conhecimento dos pais e que fazem menor número de refeições com a família (Malta et al., 2014).

Em um estudo realizado por Haugland et al. (2013), foi constatado que quando pais fazem mal uso de álcool, normalmente, seus filhos (tanto do sexo masculino, como do sexo feminino) acabam bebendo mais arriscadamente (com mais frequência, com maior intoxicação e maior quantidade). Enquanto que quando mães fazem mal uso de álcool, as meninas têm maior tendência a beber mais arriscadamente. Outro fator relevante e o segundo maior motivador do hábito de beber na adolescência é a tolerância dos pais em relação ao consumo de bebidas alcóolicas, ou seja, o quanto os pais aceitam que seus filhos consumam álcool (Wesselovicz et al., 2008). Os resultados da pesquisa de Wesselovicz et al. (2008) demonstraram que a maioria $(67,7 \%)$ dos pais dos entrevistados estavam cientes do uso da droga pelos filhos. Ademais, a percepção que os filhos têm dos pais constrói-se com base na convivência familiar diária, e esta pode ser conflituosa, quando algum dos progenitores é usuário de álcool (Lander, Howsare, \& Byerne, 2013; Wesselovicz et al., 2008).

Filhos de pais com problemas relacionados ao álcool são mais vulneráveis a vivenciar a mesma situação (Burke, Schmied, \& Montrouse, 2006; Chalder et al., 2005; Mohanan et al., 2014; Haugland et al., 2013; Oliveira, Werlang, \& Wagner, 2007; Wesselovicz et al., 2008). Isso ocorre devido a forte influência da família no comportamento de crianças e adolescentes, sendo, de grande importância a sua participação tanto na prevenção quanto no tratamento do alcoolismo (Burke, Schmied, \& Montrouse, 2006; Lander, Howsare, \& Byrne, 2013; Wesselovicz et al., 2008). O uso e abuso de álcool e outras drogas estão cada vez mais frequentes entre os adolescentes e isso pode acarretar problemas posteriores na vida do indivíduo, como a dependência de substâncias (Strauch, Pinheiro, Silva, \& Horta, 2009; Vieira, Ribeiro, Romano, \& Laranjeira, 2007). Estudos mostraram que o consumo de drogas lícitas ou ilícitas está ocorrendo com início cada vez mais precoce, com a média de idade da primeira experimentação com 11 anos (De Almeida et al., 2014; Vieira et al., 2009).

\section{OBJETIVOS E HIPÓTESE}

Este estudo teve como objetivo geral investigar qual é a relação entre o consumo de álcool dos adolescentes com a percepção deles sobre o quanto seus pais consomem bebidas alcóolicas. Também foi investigado o quanto isso afeta o consumo de álcool dos próprios jovens. Os objetivos específicos do estudo consistiram em analisar a bebida mais consumida pelos jovens e em qual idade o consumo inicia e se torna mais frequente. Como hipótese, esperou-se observar que adolescentes que percebem que os pais consomem álcool em demasia são mais propensos a também consumir (Mohanan et al., 2014; Oliveira, Werlang, \& Wagner, 2007; Haugland et al., 2013). Estudos mostraram que a bebida alcoólica mais consumida entre os adolescentes é a cerveja ou chope (Vieira et al., 2009; de Almeida et al., 2014; Carlini-Contrim, Gazal-Carvalho, \& Gouveia, 2000), esperou-se então obter o mesmo resultado.

\section{MÉTODO E INSTRUMENTO}

A amostra foi composta de 74 adolescentes (38 meninas e 36 meninos) com idades entre 10 e 16 anos de três escolas públicas e uma privada da zona sul da cidade de Porto Alegre, RS. Cinco escolas foram convidadas a participar da pesquisa (três públicas e duas privadas) e as interessadas assinaram uma autorização. Em cada um dos quatro colégios que 
autorizaram, todos os alunos dentro da faixa etária pesquisada foram convidados a participar do estudo. Foi solicitada a autorização dos pais dos adolescentes para que os mesmos participassem da pesquisa e ambos assinaram um Termo de Consentimento Livre e Esclarecido. Após, foi aplicado um questionário sobre o primeiro uso de álcool e drogas (adaptado de Galduróz, Noto, Fonseca, \& Carlini, 2004; de Almeida, et. al., 2014). Este comtempla perguntas de múltipla escolha a respeito do início e hábito do uso de álcool e drogas, questões como a frequência do uso, a idade de início, a droga ou bebida alcóolica de preferência, se algum familiar consume frequentemente álcool ou drogas, entre outras. Também contém algumas perguntas a respeito de dados sócio-econômicos.

A aplicação ocorreu nas escolas, os alunos foram convidados individualmente para realizar a avaliação em outro ambiente do colégio (em algumas escolas foi na biblioteca e em outras foi na sala de música) que não tivesse ruídos. A aplicação do questionário durou cerca de 10 minutos.

A presente pesquisa foi aprovada pelo Comitê de Ética em Pesquisa da Universidade Federal do Rio Grande do Sul, assim como está de acordo com a resolução 466/2012 do Conselho Federal de Psicologia. Este estudo faz parte de um projeto maior que engloba investigar outras questões que não serão abordadas neste artigo.

\section{ANÁLISE ESTATÍSTICA}

Primeiramente, foi analisado o consumo de álcool de forma descritiva e exploratória. Para mensurar as associações entre as variáveis categóricas, foram analisadas as tabelas de contingência usando o teste $\chi^{2}$, assim, realizou-se um teste de aderência para as variáveis dirigidas às pessoas que já tiveram experiência com bebidas alcoólicas. Também foi usado o teste exato de Fisher quando os pressupostos do $\chi^{2}$ foram violados. Foi adotado um nível de significância de 0,05 para todas as análises.

\section{RESULTADOS}

Na Tabela 1, são mostradas as frequências absolutas e relativas dos 74 adolescentes para os dados de faixa etária, tipo de escola e de relato de experiência com bebidas. Aproximadamente $40 \%$ dos adolescentes entrevistados já tiveram experiências com bebidas alcoólicas. Quase 19\% dos adolescentes entre 10 e 12 anos relataram já ter experimentado bebidas alcóolicas. A proporção de consumo de álcool aumenta conforme a idade, sendo que quase $74 \%$ dos adolescentes entre 15 e 16 anos de idade já consumiram bebidas alcoólicas. Consumo de álcool não foi significativamente associado com gênero ou o tipo de escola dos adolescentes.

TABELA 1

Frequência absoluta e porcentagem para as variáveis faixa etária, escola e padrões de consumo de álcool - N (\%).

\begin{tabular}{|c|c|c|c|}
\hline \multirow[b]{2}{*}{ Variáveis } & \multirow{2}{*}{$\begin{array}{c}\text { Participantes } \\
(n=74)\end{array}$} & \multicolumn{2}{|c|}{ Já Experimentou Álcool } \\
\hline & & $\begin{array}{c}\text { Sim } \\
(n=29)\end{array}$ & $\begin{array}{c}N \tilde{a} o \\
(n=45)\end{array}$ \\
\hline \multicolumn{4}{|l|}{ Faixa etária } \\
\hline 10-12 anos & $37(50,0)$ & $7(9,5)$ & $30(40,5)$ \\
\hline 13-14 anos & $22(29,7)$ & $11(14,9)$ & $11(14,9)$ \\
\hline 15-16 anos & $15(20,3)$ & $11(14,9)$ & $4(5,4)$ \\
\hline Gênero (feminino) & $38(51,4)$ & $13(17,6)$ & $25(33,8)$ \\
\hline \multicolumn{4}{|l|}{ Escola } \\
\hline Pública & $35(47,3)$ & $17(23,0)$ & $18(24,3)$ \\
\hline Privada & $39(52,7)$ & $12(16,2)$ & $27(36,5)$ \\
\hline
\end{tabular}

A experiência com bebidas alcoólicas também foi significativamente associada com a percepção de padrões ligados ao consumo de álcool (Tabela 2). O consumo de álcool está associado com a percepção de um consumo excessivo de bebidas alcoólicas por um familiar $\left(\chi^{2}=7,5 ; p=0,006\right)$. A figura paterna foi relatada com maior frequência a ter um consumo excessivo de álcool $\left(\chi^{2}=9,6 ; p=0,047\right.$; resíduo ajustado=2,2). Além disso, aqueles adolescentes que já experimentaram álcool relataram três vezes mais que o pai bebe excessivamente, se comparados com os adolescentes que não tiveram experiências com bebidas alcoólicas.

Foi encontrada associação estatística significativa entre considerar que alguém na família bebe excessivamente e qual familiar tem tal comportamento $\left(\chi^{2}=74 ; \mathrm{gl}=4 ; \mathrm{p}<0,001\right)$, sendo que o pai apresenta uma alta ocorrência, mas somente entre aqueles adolescentes que já tiveram experiências com bebidas alcoólicas. Ainda, entre os adolescentes que já usaram bebidas alcoólicas, as próprias casas foram o ambiente mais frequentemente relatado em que ocorreu o primeiro uso, seguido pelas casas de amigos, com um resíduo de 7,4 e 3,4; respectivamente $\left(\chi^{2}=19,8\right.$; $\mathrm{p}=0,001)$. Além disso, a vodca foi a bebida relatada como a mais consumida pelos adolescentes (com um resíduo de 4,4).

Foram testados alguns modelos para os padrões de associações mencionados acima. A relação entre o local em que houve a primeira experiência com bebidas 
alcoólicas e a percepção que um familiar bebe demais foi significativa $\left(\chi^{2}=14 ; p=0,016\right)$. Curiosamente, a área que relaciona "ter começado a beber em casa" com "considerar que um familiar bebe demais", foi justamente a que mais colaborou para a associação entre essas variáveis (resíduo ajustado $=3,4$ ). Nenhuma outra variável teve uma associação significativa com o local em que houve a primeira experiência com bebidas alcoólicas.

Outros modelos foram testados para entender a relação com o tipo de bebida mais frequentemente usada entre os adolescentes. As associações foram significativas para faixa etária $\left(\chi^{2}=26,5 ; \mathrm{gl}=10\right.$; $\mathrm{p}=0,003)$, considerar que um familiar bebe demais $\left(\chi^{2}=11,9 ; \mathrm{gl}=5 ; \mathrm{p}=0,036\right)$, com quem costuma beber $\left(\chi^{2}=86,9 ; \mathrm{gl}=10 ; \mathrm{p}<0,001\right)$. No entanto, essa associação foi, em grande parte, devido ao consumo elevado de vodca pelos adolescentes. A vodca foi citada frequentemente entre os adolescentes na faixa etária entre 14 e 16 anos. A bebida foi mais frequente que o esperado entre aqueles adolescentes que consideram que um familiar bebe demais. A vodca também está altamente associada ao consumo na presença de amigos.

Além disso, foi testada a associação entre o relato da companhia que os adolescentes costumam consumir bebidas alcoólicas e faixa etária. Houve uma associação significativa entre essas variáveis que pode ser observada na tabela $2\left(\chi^{2}=21,3 ; \mathrm{gl}=4 ; \mathrm{p}<0,001\right)$. Dada a frequência observada para o grupo na faixa etária entre 14 e 16 anos, pode-se inferir que apenas entre os adolescentes mais velhos os amigos passam a ter um papel determinante no padrão e frequência de consumo de bebidas alcoólicas. No grupo entre 12 e 14 anos, o consumo de bebidas com os familiares é mais frequentemente relatado do que os valores esperados pelo modelo, o que nos permite inferir que o grupo familiar exerce uma influência significativa no consumo mais precoce de bebidas alcoólicas. Cabe salientar que o relato de qual membro da família bebe demais não foi significativamente associado com gênero, faixa etária ou tipo de escola.

\section{DISCUSSÃO}

A vodca foi a bebida relatada como mais consumida entre os adolescentes na amostra estudada, sendo que o estudo de Ballistreri e Corradi-Webster (2008) mostrou como resultado que as bebidas energéticas são bastante consumidas com essa combinação e, a mesma, tem-se mostrado mais frequente no consumo por jovens. Estudos mostraram que as bebidas mais consumidas pelos adolescentes são o chope e a vodca
(Centers for Disease Control and Prevention, 2009; Locatelli, Sanchez, Opaleye, Carlini, \& Noto, 2012; Wilczyński, Witowski, Pawlik, Krysta, \& KrupkaMatuszczyk, 2013) e esta última está ganhando cada vez mais espaço entre os jovens. $\mathrm{O}$ consumo de tal destilado pode estar associado ao baixo custo para venda, além de apresentar alto teor alcoólico e sabor camuflado pelos gostos adocicados dos componentes que são misturados à bebida, por exemplo, mistura de vodca com energético, suco ou refrigerantes (Giordano \& Giordano, 2009). Estudo de De Almeida et al. (2014) encontrou como resultado a cerveja como bebida alcóolica mais consumida entre os jovens, os resultados do presente estudo refutam a hipótese inicial, que obteve como resultado a vodca como bebida alcóolica mais consumida entre os adolescentes.

Um estudo encontrou como resultado a média de 16,82 anos como a idade do primeiro consumo de álcool (Mohanan, et. al., 2014). O presente estudo encontrou resultados semelhantes, na medida em que $74 \%$ dos adolescentes de 15 e 16 anos declararam já ter experimentado algum tipo de bebida alcoólica. Este dado associado com o estudo de Mohanan et al. (2014) sugere que grande parte dos jovens consomem álcool após os 15 anos, idade esta que o consumo da bebida alcóolica ainda é ilegal no Brasil. Isso mostra que os adolescentes têm acesso à droga de alguma forma, mesmo que a comercialização e distribuição de bebidas alcoólicas para menores de 18 idade seja proibida (Lei no 8069 de 1990 do Estatuto da Criança e do Adolescente).

No presente estudo pôde-se observar que há um aumento no percentual do primeiro consumo de álcool conforme se chega mais próximo da idade referida acima. Menos de 20\% dos estudantes entre 10 e 12 anos já experimentaram álcool. Esse número chega aos $50 \%$ entre 13 e 14 anos e aos $74 \%$ entre 15 e 16 anos. Os adolescentes apresentaram consumo de álcool de forma precoce e outros estudos demonstraram que os jovens começam a experimentar tal droga com 12 anos de idade (Vieira et al., 2009; De Almeida et al., 2014), idade a qual muitos sequer chegaram à puberdade.

Diversos estudos se propõem a investigar o consumo excessivo de álcool em escolas da rede pública (Anjos, Santos, \& Almeida, 2012; Cerqueira et al., 2011; Gomes, Alves, Aquino, Medeiros, \& Lima, 2014; Gomes, Alves, \& Nascimento, 2010), no entanto, não são muitos os que comparam este uso de bebida alcóolica entre adolescentes de escolas públicas e privadas. Um estudo transversal em São Paulo com 1025 adolescentes mostrou que uso de drogas ilícitas é de maior parte da burguesia e o uso de álcool é 
maior no proletariado (Muza, Bettiol, Muccillo, \& Barbieri, 1997). Uma pesquisa realizada em Pelotas, RS não encontrou diferenças significativas entre o uso de bebida alcóolica e tipo de escola (Tavares,
Béria, \& Lima, 2001), o presente estudo obteve o mesmo resultado, não foram encontradas diferenças significativas do consumo de álcool entre escola pública e privada.

TABELA 2

Associação entre as variáveis categóricas e já ter experimentado bebidas alcoólicas entre adolescentes medidas através do teste $\chi^{2}$ de Pearson ou teste exato de Fisher.

\begin{tabular}{|c|c|c|c|c|c|}
\hline \multirow{2}{*}{ Variáveis } & \multicolumn{2}{|c|}{ Já Experimentou Álcool } & \multirow{2}{*}{$\chi^{2} / F^{a}$} & \multirow{2}{*}{$g l$} & \multirow{2}{*}{$p$} \\
\hline & Sim & Não & & & \\
\hline Faixa Etária & & & 14,786 & 2 & 0,001 \\
\hline $10-12$ anos & 7 & 30 & & & \\
\hline 13-14 anos & 11 & 11 & & & \\
\hline $15-16$ anos & 11 & 4 & & & \\
\hline Gênero & & & 0,812 & 1 & 0,367 \\
\hline Feminino & 13 & 25 & & & \\
\hline Masculino & 16 & 20 & & & \\
\hline Escola & & & 2,453 & 1 & 0,117 \\
\hline Pública & 17 & 18 & & & \\
\hline Privada & 12 & 27 & & & \\
\hline Acredita que alguém familiar bebe demais? & & & 7,536 & 1 & 0,006 \\
\hline Sim & 12 & 6 & & & \\
\hline Não & 17 & 39 & & & \\
\hline Quem você considera que beba demais na sua família? & & & 9,634 & 4 & 0,47 \\
\hline Não & 17 & 39 & & & \\
\hline Pai & 6 & 2 & & & \\
\hline Mãe & 0 & 1 & & & \\
\hline Irmãos & 2 & 1 & & & \\
\hline Outros & 4 & 2 & & & \\
\hline Onde você consumiu álcool pela primeira vez? & & & 19,857 & 4 & 0,001 \\
\hline Em Casa & 13 & & & & \\
\hline Bares ou similares & 4 & & & & \\
\hline Casa de amigos & 9 & & & & \\
\hline Não lembro & 1 & & & & \\
\hline Outros & 1 & & & & \\
\hline Qual bebida alcoólica você consome com mais frequência? & & & 25,929 & 4 & $<0,001$ \\
\hline Não costumo beber & 14 & & & & \\
\hline Cerveja ou Chopp & 2 & & & & \\
\hline Cachaça & 1 & & & & \\
\hline Vodca & 10 & & & & \\
\hline Outro & 1 & & & & \\
\hline Com quem você costuma beber com mais frequência? & & & 5,643 & 2 & 0,060 \\
\hline Não costumo beber & 8 & & & & \\
\hline Familiares & 5 & & & & \\
\hline Amigos & 15 & & & & \\
\hline
\end{tabular}

a. Teste exato de Fisher. 
O papel dos pais na vida dos adolescentes é crucial para o desenvolvimento e influencia diversos campos da vida dos filhos, incluído o uso de álcool e outras drogas (Pechansky, Szobot, \& Scivoletto, 2004). O consumo de drogas pelos pais ou pelos irmãos e a atitude parental permissiva com relação ao uso de álcool e drogas também são apontadas como predisponentes a iniciação ou continuação do uso de bebidas alcóolicas e outras drogas pelos adolescentes (Pechansky, Szobot, \& Scivoletto, 2004; Brooke \& Brooke, 1996).

Foi possível observar nos resultados que houve relação significativa entre considerar que um familiar bebe excessivamente e o consumo do álcool pelo adolescente. Um dos fatores de risco para o uso em demasia da bebida alcoólica pelos jovens é o ambiente doméstico, principalmente relacionado a pais abusadores de alguma substância e a paternidade não participante na vida dos filhos (Pechansky, Szobot, \& Scivoletto, 2004; Souza, Areco, Da Silveira Filho, 2005).

Há uma associação positiva entre hábitos de consumo de álcool dos pais e daqueles que os rodeiam, incluindo seus filhos (Mohanan, 2014, Chalder et al., 2005; Oliveira, Werlang, \& Wagner, 2007), e isso devese ao fato do comportamento dos progenitores ser um importante fator ambiental (Haugland, et al., 2013). Em estudo de Mohanan (2014), 54,5\% dos sujeitos consumidores de álcool revelaram que pelo menos um de seus parentes tinham problemas com a bebida. Chalder et al. (2005) verificou uma maior frequência e quantidade no consumo de álcool pelos filhos de pais com problemas relacionados à droga. $\mathrm{E}$ os resultados do presente estudo corroboram tais dados.

Um estudo mostrou que o uso excessivo de álcool é mais comum pelos pais do que pelas mães (Haugland, et al., 2013). O mesmo resultado foi constatado no presente estudo, que verificou que dentre os sujeitos que declararam já ter consumido álcool e possuir algum parente com hábitos alcoólatras, $50 \%$ atribuíram tal excesso ao pai.

A maioria dos jovens que acredita ter algum familiar que beba demais já experimentou álcool (66,6\% dos que percebem que algum familiar bebe excessivamente). E, assim como em Haugland et al. (2013), foi encontrado no presente estudo que o fato de o pai beber demais é mais influente no início do uso do álcool que o de a mãe beber. No estudo de Haugland et al. (2013), infere-se inclusive que ter mães que bebam em demasia diminui a chance de adolescentes consumirem altos níveis de álcool. Os motivos de tais achados continuam sem explicação, assim, estudos futuros são necessários para uma melhor compreensão de tais resultados.
Encontrou-se relação entre o local de primeira experiência, que foi a própria casa, e a percepção de consumo exacerbado de álcool por algum integrante da família. Esse dado pode estar associado com a aceitabilidade dos pais, que em sua maioria são cientes e permissivos ao uso de álcool pelos filhos (Wesselovicz et al., 2008), podendo, assim, oferecerlhes no ambiente familiar.

Apesar do consumo de álcool iniciar com os pais, os jovens bebem com mais frequência na presença de amigos, pois adolescentes estão mais suscetíveis a ser influenciados pelo grupo de amizade, além de estarem mais propensos a ter comportamentos de risco em grupo do que quando estão sozinhos (Loke \& Mak, 2013; Pechansky, Szobot, \& Scivoletto, 2004).

Os adolescentes que consideram que seus pais consomem álcool em demasia, experimentaram bebidas alcoólicas mais precocemente. Além disso, consumiram álcool mais frequentemente $\mathrm{e}$ intensamente, quando comparados com adolescentes que não tenham considerado que seus pais ingiram bebidas alcóolicas em grande quantidade. Os jovens que possuem familiares que abusam do álcool também consomem bebidas mais fortes e com mais percentual de álcool. Pode-se concluir que a imagem que os filhos têm a respeito do consumo de álcool dos seus pais, influencia diretamente o próprio consumo.

Um estudo que investiga a venda de bebidas alcoólicas para menores de 18 anos $(n=534)$ no estado de São Paulo obteve como resultado que mais de $80 \%$ dos adolescentes conseguiram comprar bebida alcóolica e ressaltou a importância de adiar o consumo de álcool do início da adolescência para seu final e constituir esforços de prevenção (Romano, Duailibi, Pinsky, \& Laranjeira, 2007). O estudo que se propôs a adaptar e validar o Alcohol Use Disorders Identification Test (AUDIT) para a população do interior do Amazonas, identificou que o teste permite um enfoque na prevenção, uma vez que o paciente é sensibilizado para a redução do uso de álcool (MorettiPires \& Corradi-Webster, 2011).

É importante salientar estudos que mantenham o foco na prevenção do uso de álcool na adolescência, visto que esse uso pode trazer malefício a curto, médio e longo prazo para a vida de quem consome. Também torna-se importante ressaltar que este estudo possui uma amostra pequena por conveniência, por tanto não pode ser replicada, o que é uma limitação da pesquisa. Apesar de o instrumento utilizado já ter sido adaptado e utilizado em outro estudo (De Almeida et al., 2014), o uso de mais questionários ou uma bateria mais completa, beneficiaria em resultados mais sólidos. Se espera que este estudo possa ser replicado 
em outras amostras brasileiras para que os resultados possam trazer maior avanço para a área, a fim de se compreender essa problemática do uso excessivo de bebida alcóolica na adolescência.

\section{CONSIDERAÇÕES FINAIS}

Observou-se nesta pesquisa que os jovens têm provado bebidas alcoólicas precocemente, ao redor dos 12 anos, e $40 \%$ da amostra já consumiu ou experimentou álcool pelo menos uma vez. Além disso, este estudo avaliou a bebida consumida em maior frequência, o que resultou no relato de adolescentes consumirem, em sua maioria, vodca, o que diferiu de diversos estudos que relataram que os adolescentes consomem mais cerveja. Isso pôde ser observado porque o consumo da vodca está cada vez mais recorrente por se misturar com bebidas mais doces como o energético.
O uso de bebida alcoólica está fortemente associado com a percepção que os jovens têm do abuso dessa substância por parte dos pais. Os adolescentes que consomem álcool em maiores quantidades ou ingerem bebidas com maior percentual alcoólico consideram que algum familiar bebe em demasia, sendo em maior recorrência o pai. O papel dos pais no desenvolvimento dos adolescentes é essencial e pode influenciar o início ou a continuidade do consumo de álcool na adolescência, pois jovens que tem pais os quais consomem ou que não desaprovam o consumo tendem a beber mais e iniciar o consumo de álcool mais precocemente. Sobretudo, mais pesquisas neste campo são necessárias para que se possa alertar a sociedade e os pais de adolescentes sobre o consumo de drogas, incluindo o álcool na adolescência, período demarcado por mudanças importantes as quais estão sob influência de diversos fatores, incluindo comportamento parental.

\section{REFERÊNCIAS}

Anjos, K. F., Santos, V. C., \& Almeida, O. S. (2012). Caracterização do consumo de álcool entre estudantes do ensino médio. Revista Baiana de Saúde Pública, 36(2), 418-431. Disponível em: <http://inseer.ibict.br/rbsp/index.php/rbsp/ article/viewFile/468/pdf_149>.

Ballistreri, M. C., \& Corradi-Webster, C. M. (2008). Consumption of energy drinks among physical education students. Rev Latino-am Enfermagem, 16(especial), 558-64. http://dx.doi.org/10.1590/S0104-11692008000700009

Benchaya, M. C., Bisch, N. K., Moreira, T. C., Ferigolo, M., \& Barros, H. M. T. (2011). Non-authoritative parents and impact on drug use: the perceptions of adolescent children. Jornal de Pediatria, 87(3), 238-244. http://dx.doi. org/10.2223/JPED.2089

Brook, J. S., \& Brook, D. W. (1996). Risk and protective factors for drug use. In Mcoy, C., Metsch, L. K., \& Inciardi, J. A (Eds.). Intervening with drug-involved youth (pp. 23-43). Sage Publications.

Burke, S., Schimied, V., \& Montrose, M. (2006). Parental alcohol misuse and the impact on children-A literature review. Ashfield: Department of Community Services, NSW. pp. 5-15.

Carlini-Contrim, B., Gazal-Carvalho, C., \& Gouveia, N. (2000). Comportamentos de saúde entre jovens estudantes das redes pública e privada da área metropolitana do Estado de São Paulo. Revista de Saúde Pública, 34(6), 636-645. Recuperado de: <http://www.scielosp.org/pdf/rsp/v34n6/3579>.

Centers for Disease Control and Prevention. (2009). Alcohol use among high school students - Georgia, 2007. MMWR Morb Mortal Wkly Rep., 58(32), 885-90. Recuperado em: 21 jul. 2014, disponível em: <http://www.cdc.gov/mmwr/ preview/mmwrhtml/mm5832a1.htm>.

Cerqueira, G., Lucena, C., Gomes, A., Freitas, A., Rocha, N., \& Mariz, S. (2011). Consumo de álcool entre estudantes de uma escola pública da cidade de Cajazeiras, PB. SMAD - Revista Eletrônica Saúde Mental Álcool e Drogas (Edição em Português), 7(1), 18-24. http://dx.doi.org/10.11606/issn.1806-6976.v7i1p18-24

Chalder, M., Elgar, F. J., \& Bennet, P. (2005). Drinking and Motivation to Drink Among Adolescent Children of Parents with Alcohol Problems. Alcohol And Alcoholism, Winnipeg, 41(1), 107-113. http://dx.doi.org/10.1093/alcalc/ $\operatorname{agh} 215$

Crone, E. A. (2009). Executive functions in adolescence: inferences from brain and behavior. Developmental Science 12(6), 825-830.

De Almeida, R. M. M., Trentini, L. B., Klein, L. A., Macuglia, G. R., Hammer, C., \& Tesmmer, M. (2014). Uso de Álcool, Drogas, Níveis de Impulsividade e Agressividade em Adolescentes do Rio Grande do Sul. Psico, 45(1), 65-72. Recuperado em: 21 jul. 2014, disponível em: <http://revistaseletronicas.pucrs.br/revistapsico/ojs/index.php/ revistapsico/article/view/12727>. http://dx.doi.org/10.15448/1980-8623.2014.1.12727

Giordano, M. V., \& Giordano, L. A. (2009). Contracepção na adolescência. Adolescência \& saúde, 6(4), 11-16. Recuperado em: 14 jan. 2015, disponível em: <http://adolescenciaesaude.com/detalhe_artigo.asp?id=4>. 
Gomes, B. M. R., Alves, J. G. B., Aquino, J. M., Medeiros, S. E. G., \& Lima, F. M. (2014). Fatores associados ao consumo de álcool entre estudantes de escolas públicas. Revista de Enfermagem UFPE, 8(5), 1164-1170. http://dx.doi. org/10.5205/reuol.5863-50531-1-ED.0805201409

Gomes, B. M. R., Alves, J. G. B., \& Nascimento, L. C. (2010). Consumo de álcool entre estudantes de escolas públicas da Região Metropolitana do Recife, Pernambuco, Brasil. Cadernos de Saúde Pública, 26(4), 706-712. http://dx.doi. org/10.1590/S0102-311X2010000400013

Galduróz, J. C. F., Noto, A. R., Nappo, S. A., \& Carlini, E. A. (2004). Trends in drug use among students in Brazil: analysis of four surveys in 1987, 1989, 1993 and 1997. Brazilian Journal of Medical and Biological Research, 37(4), 523-531. http://dx.doi.org/10.1590/S0100-879X2004000400009

Gullo, M. J. \& Dawe, S. (2008). Impulsivity and adolescent substance use: Rashly dismissed as "all-bad"? Neurosci Biobehav Rev, 32, 1507-1518. http://dx.doi.org/10.1016/j.neubiorev.2008.06.003

Haugland, S. H., Holmen, T. L., Ravndal, E., \& Bratberg, G. H. (2013). Parental alcohol misuse and hazardous drinking among offspring in a general teenage population: gender-specific findings from the Young-HUNT 3 study. Biomed Central: Public Health, 13(1140). http://dx.doi.org/10.1186/1471-2458-13-1140

Janssen, M. M., Mathijssen, J. J. P., Bon-Martens, M. J. H., Oers, H. A. M., \& Garretsen, H. F. L. (2012). A qualitative exploration of attitudes towards alcohol, and the role of parents and peers of two alcohol-attitude-based segments of the adolescent population. Substance Abuse Treatment, Prevention, and Policy, 9(20). http://dx.doi.org/10.1186/1747$597 \mathrm{X}-9-20$

Lander, L., Howsare, J., \& Byrne, M. (2013). The Impact of Substance Use Disorders on Families and Children: From Theory to Practice. Social Work Public Health, Morgantown, 28(0), 194-205. http://dx.doi.org/10.1080/19371918.2 013.759005

Locatelli, D., Sanchez, Z., Opaleye, E., Carlini, C., \& Noto, A. (2012). Socioeconomic influences on alcohol use patterns among private school students in São Paulo. Revista Brasileira de Psiquiatria, 34(2). http://dx.doi.org/10.1590/S151644462012000200012

Loke, A. Y. \& Mak, Y. (2013). Family Process and Peer Influences on Substance Use by Adolescents. International Journal Of Environmental Research And Public Health 10(9), 3868-3885. http://dx.doi.org/10.3390/ijerph 10093868

Looze, M., Vermeulen-Smit, E., Bogt, T. F. M., Dorsselaer, S. A. F. M., Verdumen, J. Schulten, I., Engels, R. C. M. E., \& Vollebergh, W. A. M. (2014). Trends in alcohol-specific parenting practices and adolescent alcohol use between 2007 and 2011 in the Netherlands. International Journal Of Drug Policy, 25(1), 133-141. http://dx.doi.org/10.1016/j. drugpo.2013.09.007

Malta, D. C., Mascarenhas, M. D. M., Porto, D. L., Barreto, S. M., \& Neto, O. L. M. (2014). Exposição ao álcool entre escolares e fatores associados. Revista de Saúde Pública, 48(1), 52-62. http://dx.doi.org/10.1590/S00348910.2014048004563

Mohanan, P., Swain, S., Sanah, N., Sharma, V., \& Ghosh, D. (2014). A Study on the Prevalence of Alcohol Consumption, Tobacco Use and Sexual Behaviour among Adolescents in Urban Areas of the Udupi District, Karnataka, India. Sultan Qaboos Univ Med J., 14(1), 104-112. Recuperado em: 21 jul. 2014, disponível em: <http://www.ncbi.nlm.nih.gov/ pmc/articles/PMC3916261/>. http://dx.doi.org/10.12816/0003343

Moretti-Pires, R. O. \& Corradi-Webster, C. M. (2011). Adaptação e validação do Alcohol Use Disorder Identification Test (AUDIT) para população ribeirinha do interior da Amazônia, Brasil. Cad. saúde pública, 27(3), 497-509. http:// dx.doi.org/10.1590/S0102-311X2011000300010

Muza, G. M., Bettiol, H., Muccillo, G., \& Barbieri, M. A. (1997). Consumo de substâncias psicoativas por adolescentes escolares de Ribeirão Preto, SP (Brasil). Revista de Saúde Pública, 31, 163-170. http://dx.doi.org/10.1590/S003489101997000100005

Newman, K., Harrison, L., Dashiff, C., \& Davies, S. (2008). Relações entre modelos de pais e comportamentos de risco na saúde do adolescente: uma revisão integrativa da literatura. Revista Latino-Americana de Enfermagem, 16(1), 142-150. http://dx.doi.org/10.1590/S0104-11692008000100022

Oliveira, M. da S., Werlang, B. S. G., \& Wagner, M. F. (2007). Relação Entre o Consumo de Álcool e Hábitos Paternos de Ingestão de Alcóolica. Boletim de Psicologia, 57(127), 205-214. Recuperado em: 21 jul. 2014, disponível em: $<$ http://pepsic.bvsalud.org/pdf/bolpsi/v57n127/v57n127a07.pdf>.

Pechansky, F., Szobot, C. M., \& Scivoletto, S. (2004). Uso de álcool entre adolescentes: conceitos, características epidemiológicas e fatores etiopatogênicos. Revista Brasileira de Psiquiatria, 26(Suppl. 1), 14-17. http://dx.doi. org/10.1590/S1516-44462004000500005

Roehrs, H., Lenardt, M. H., \& Maftum, M. A. Práticas culturais familiares e o uso de drogas psicoativas pelos adolescentes: reflexão teórica. Escola Anna Nery Revista de Enfermagem, 12(2), 353-357. Recuperado em: 21 jul. 2014, disponível em: <http://www.scielo.br/pdf/ean/v12n2/v12n2a24.pdf $>$.

Romano, M., Duailibi, S., Pinsky, I., \& Laranjeira, R. (2007). Pesquisa de compra de bebidas alcoólicas por adolescentes em duas cidades do Estado de São Paulo. Revista de saúde publica, 41(4), 495-501. http://dx.doi.org/10.1590/S003489102007000400001 
Shirtcliff, E. A., Dahl, R. E., \& Pollak, S. D. (2009). Pubertal development: correspondence between hormonal and physical development. Child Development, 80(2), 327-337. http://dx.doi.org/10.1111/j.1467-8624.2009.01263.x

Skinner, E., Johnson, S., Snyder, T. (2005). Six dimensions of parenting: a motivational model. Parent Sci Pract., 5(2), 175-235. Recuperado em: 21 jul. 2014, disponível em: <http://selfdeterminationtheory.org/SDT/documents/2005 SkinnerJohnsonSnyder_PASP.pdf>. http://dx.doi.org/10.1207/s15327922par0502_3.

Souza, D. P. O., Areco, K. N., \& da Silveira Filho, D. X. (2005). Álcool e alcoolismo entre adolescentes da rede estadual de ensino de Cuiabá, Mato Grosso. Revista de saúde Pública, 39(4), 585-592.

Strauch, E. S. Pinheiro, R. T., Silva, R. A., \& Horta, B. L. (2009). Uso de álcool por adolescentes: estudo de base populacional. Revista de Saúde Pública, 43(4), 647-655. http://dx.doi.org/10.1590/S0034-89102009005000044

Tavares, B. F., Béria, J. U., \& Lima, M. S. (2001). Prevalência do uso de drogas e desempenho escolar entre adolescentes. Revista de Saúde Pública, 35(2), 150-8. Disponível em: <http://www.scielo.br/pdf/rsp/v35n2/4399.pdf>. http://dx.doi. org/10.1590/S0034-89102001000200008

Verdejo-Garcia, A.; Lawrence, A. J., \& Clark, L. (2008). Impulsivity as a vulnerability marker for substance-use disorders: Review of findings from high-risk research, problem gamblers and genetic association studies. Neurosci Biobehav Rev., 32, 777-810. http://dx.doi.org/10.1016/j.neubiorev.2007.11.003

Wesselovicz, A. A. G., Sousa, T. G., Kaneshima, E. N., \& Sousa-Kaneshima, A. M. (2008). Fatores associados ao consumo de bebidas alcóolicas pelos adolescentes de uma Escola Pública da cidade de Maringá, Estado do Paraná. Acta Scientiarum. Health Science, 30(2), 161-166. http://dx.doi.org/10.4025/actascihealthsci.v30i2.917

Wilczyński, K., Witowski, Ł., Pawlik, A., Krysta, K., \& Krupka-Matuszczyk, I. (2013). Consumption of alcohol and risk of alcohol addiction among students in Poland. Psychiatr Danub., 25(Suppl 2), S78-82. Recuperado em: 21 jul. 2014, disponível em: <http://www.ncbi.nlm.nih.gov/pubmed/23995150>.

Vieira, D. L., Ribeiro, M., Romano, M., \& Laranjeira, R. R. (2007). Alcohol and adolescents: study to implement municipal policies. Revista Saúde Pública, 41(3), 396-403. http://dx.doi.org/10.1590/S0034-89102006005000022

\footnotetext{
Autores:

Endereço para correspondência:

Rosa Maria Martins de Almeida

Universidade Federal do Rio Grande do $\mathrm{Su}$

Rua Ramiro Barcelos, 2600 - Bairro Santa Cecília

CEP 90035-003 Porto Alegre, RS, Brasil

E-mail: rosa almeida@yahoo.com rosa.almeida@ufrgs.br

Recebido em: 21.07.14

Aceito em: $16.01 .15 \mathrm{z}$
}

Alice Rodrigues Willhelm - Mestranda, Universidade Federal do Rio Grande do Sul JoÃo CARlos CEBtURION CABRAL - Mestrando, Universidade Federal do Rio Grande do Sul. Luiza Mugnol UGARTE - Mestranda, Universidade Federal do Rio Grande do Sul.

JANAÍNA OLIVEIRA STEIGER - Iniciação Ciêntífica, Universidade Federal do Rio Grande do Sul. José FELIPE Flores DA SILVA - Iniciação Ciêntífica, Universidade Federal do Rio Grande do Sul. Rosa MARIA MARTINS DE AlmEIDA - Doutora, Universidade Federal do Rio Grande do Sul. 